\title{
B-type natriuretic peptide: Usefulness in the management of critically-ill neonates
}

\author{
Gisela L. Salas, M.D. ${ }^{a}$, Mariela Jozefkowicz, M.D. ${ }^{a}$, Gustavo S. Goldsmit, M.D. ${ }^{a}$, \\ Gabriela Disa, Biochemist ${ }^{b}$, Alejandra Rodiño, Biochemist ${ }^{b}$, Susana Rodríguez, M.D. ${ }^{c}$ \\ and Diana Fariña, M.D. ${ }^{a}$
}

\begin{abstract}
B-type natriuretic peptide (BNP) is responsible for changes in the heart organogenesis and is associated with transition toextrauterinelife. In the first week of life, BNP levels are high and return to normal with the physiological loss in weight. High BNPlevels are associated with different conditions. Objective. To establish the relationship between BNP levels and criticality and the short-term clinical course among patients hospitalized in the neonatal intensive care unit.

Method. Observational and analytical study conducted in a prospective cohort. Criticality was defined as a requirement for assisted mechanical ventilation with a fraction of inspired oxygen of more than $50 \%$ and / or inotropes. Two blood samples were collected 72 hours apart.

Results. Seventy-three patients were included in the study. Depending on their clinical course on day 7 , they were divided into 2 groups: patients with a good clinical course or a persistent, severe clinical course. The median baseline BNP level was similar in both groups ( $p: 0.15)$. The median BNP level at 72 hours was higher among patients with a persistent, severe clinical course ( $p: 0.005)$. The difference between both BNP values was calculated ( $\triangle \mathrm{BNP}$ : BNP level at 72 hours - BNP level at 0 hours). The $\triangle B N P$ was positive among patients with a persistent, severe clinical course $(X=1260 \mathrm{pg} / \mathrm{mL}$; range: $426-2094)$ and negative in the group with a good clinical course $(X=-967$ $\mathrm{pg} / \mathrm{mL}$; range:-1656/-278) ( $p: 0.0002)$; sensitivity: $87 \%$; specificity: $86 \%$; positive predictive value: $74 \%$; and negative predictive value: $93 \%$.

Conclusion. In this group of patients, the deltaBNP value reflected the short-term clinical course.

Key words: B-type natriuretic peptide, neonates.
\end{abstract}

http:/ / dx.doi.org/10.5546/ aap.2017.eng.483

To cite: Salas G, Jozefkowicz M, Goldsmit GS, et al. B-type natriuretic peptide: Usefulness in the management of critically-ill neonates. Arch Argent Pediatr 2017;115(5):483-489.
Funding:

Research fellowship granted by Hospital de Pediatría "Prof. Dr. Juan P. Garrahan".

Conflict of interest:

None.

Received: 10-20-2016

Accepted: 3-30-2017

\section{INTRODUCTION}

B-type natriuretic peptide (BNP) is a cardiac peptide released by the heart ventricles in response to changes in the ventricular pressure and/or volume. ${ }^{1,2}$

BNP is a peptide made up of 32 amino acids with a ring structure; its mechanism of action is mediated by the increase in cyclic guanosine monophosphate (cGMP). It has a diuretic and pulmonary vasodilatory effect. BNP plays a major role in the organogenesis of the fetal heart and during the transition of newborn infants (NBIs) to extrauterine life. ${ }^{3,4}$

In the first week of life, BNP levels are normally high, up to 12 times higher than maternal levels, since it does not cross the placental barrier. Such high value observed among healthy NBIs in the first days of life tends to return to normal with physiological loss in weight reaching a level similar to that observed in adults. ${ }^{5-7}$

In the neonatal population, BNP measurements vary widely. Several studies have reported BNP levels ranging from 200 to $6000 \mathrm{pg} / \mathrm{mL}$. Following the first week, BNP levels tend to normalize and can even be lower than $300 \mathrm{pg} / \mathrm{mL}{ }^{8}$

In the adult population, there is evidence that an increase in BNP and its N-terminal fraction (NT-proBNP) is associated with heart failure $^{9,10}$ and an increase in mortality due to severe sepsis and septic shock. ${ }^{11}$

In the pediatric population, a dramatic rise in BNP and NTproBNP levels has been associated with hemodynamic compromise, congenital heart malformations, and a poor clinical course. ${ }^{12-14}$ 
For patients with pulmonary hypertension (PHT), BNP has demonstrated to be a useful prognostic marker. Changes in BNP levels during the longitudinal follow-up of a patient appear to be more important than an isolated measurement, even if it was high. ${ }^{15}$

Among preterm NBIs, elevated BNP and NTproBNP levels have been associated with the presence of patent ductus arteriosus (PDA) ${ }^{16,17}$ and the severity of hemodynamic compromise. ${ }^{18,19}$ Such high levels correlate with the size of the PDA and the increase in pulmonary blood flow. ${ }^{20,21}$

To date, no reports have been published on the assessment of this marker as a predictor of clinical course among sick neonates or on whether there is an association between BNP levels and/or an increase in BNP levels and the lack of stabilization among critically-ill NBIs.

The hypothesis posed by this study was that BNP levels were higher among critically-ill NBIs and that a decrease in BNP levels was related to a good clinical course (GCC) and treatment response. BNP measurement may help to make decisions regarding the timely transfer of patients in this subgroup to tertiary care (level III) hospitals, in advance of their need for more complex therapeutic support.

The objective of this study was to establish whether there is a relationship between BNP levels and/or variations and criticality and the short-term clinical course among patients with cardiorespiratory instability hospitalized in the neonatal intensive care unit (NICU).

\section{METHOD}

This was an observational and analytical study conducted in a prospective cohort. All neonates who met the criteria for hemodynamic instability and admitted to the NICU were included in the study consecutively and only once.

The study was conducted in the Neonatal Intensive Care Unit of Hospital Nacional de Pediatría SAMIC "Prof. Dr. Juan P. Garrahan", a tertiary care (level IIIB) hospital, in the period between July $1^{\text {st }}, 2013$ and June $30^{\text {th }}, 2014$.

Inclusion criteria were as follows: patients with hemodynamic instability, defined as requiring assisted mechanical ventilation (AMV) with a fraction of inspired oxygen $\left(\mathrm{FIO}_{2}\right)$ of more than $50 \%$ or as requiring AMV, with any level of $\mathrm{FIO}_{2}$, plus inotropes.

Neonates born at less than 35 weeks' gestation, patients with malformations incompatible with life, and those for whom informed consent was not obtained were excluded.
All patients who died before 72 hours of life were removed.

Two blood samples were obtained from all patients: the first one at inclusion in the study and the second one, 72 hours later.

Samples consisted in $1.3 \mathrm{~mL}$ of blood collected in ethylenediaminetetraacetic acid (EDTA) tubes, which were centrifuged immediately for plasma BNP measurement. Measurements were made by microparticle enzyme immunoassay (AXSYM system, ABBOTT laboratory) and values were reported as $\mathrm{pg} / \mathrm{mL}$.

Additionally, baseline and clinical course data were collected over 7 days after inclusion in the study.

Study outcome measures were age in days, sex, gestational age, birth weight, Apgar score, delivery mode, referral requirement, diagnosis, Score for Neonatal Acute Physiology-Perinatal Extension (SNAPPE) on admission. Criticality and clinical course were established based on AMV requirement, mean airway pressure (MAP), $\mathrm{FIO}_{2}$, oxygenation index (OI), inotrope use, vasoactive-inotropic score (VIS) ${ }^{22}$ (dose: dopamine + dobutamine $+100 \times$ epinephrine $+10 \mathrm{x}$ milrinone $+10000 \mathrm{x}$ vasopressin $+100 \mathrm{x}$ norepinephrine), inhaled nitric oxide (iNO) use, extracorporeal membrane oxygenation (ECMO) requirement, impairment of liver and/or kidney function, multiple organ dysfunction syndrome (MODS), and death.

Based on their clinical condition on day 7, patients were divided into 2 groups to establish the primary outcome measure: patients with a good clinical course or patients with a persistent severe clinical course (PSCC), defined as cardiorespiratory instability in spite of treatment (AMV and inotrope requirement that is the same or higher than at study inclusion, ECMO requirement or death).

The $\triangle \mathrm{BNP}$ was measured to analyze the role of marker variations. It was calculated as the difference between the BNP level at 72 hours minus the BNP level at admission, for each patient.

The protocol was assessed and approved by the Review Committee and the Ethics Committee of Hospital Nacional de Pediatría SAMIC "Prof. Dr. Juan P. Garrahan". An informed consent for study participation was obtained from patients families.

\section{Statistical analysis}

All outcome measures were summarized using descriptive statistics of central tendency, 
position, and dispersion.

The sample size was estimated to establish a $500 \mathrm{pg}$ difference with a more/less $500 \mathrm{pg}$ variability; considering an 0.05 alpha error and an 0.10 beta error, 22 children were required in each arm (patients with GCC and PSCC).

BNP levels at baseline and at 72 hours were described as median and interquartile range.

To measure marker variations, the $\triangle \mathrm{BNP}$ was defined as the difference between the second BNP value minus the first $B N P$ value for each patient.

Patients' BNP levels, $\triangle \mathrm{BNP}$, and clinical course (GCC versus PSCC) were compared using a Wilcoxon non-parametric test. A value of $p<0.05$ was considered statistically significant. Categorical outcome measures were compared using the $\chi^{2}$ test.

A non-parametric receiver operating characteristic (ROC) curve was developed to establish the cut-off point at which the highest sensitivity and specificity were achieved and to assess its discrimination power as diagnostic test, i.e., its capability to differentiate GCC from PSCC. The area under the curve and the $95 \%$ confidence interval (CI), as well as sensitivity, specificity, positive predictive value (PPV), and negative predictive value (NPV), were estimated for said cut-off value, which was selected based on the Youden index.

The statistical analysis was performed with the STATA SE 12.0 software (StataCorp LP, USA).

\section{RESULTS}

Seventy-three critically-ill patients were included in the study (Figure 1).

The general characteristics of patients are summarized in Table 1.

As shown in Table 2, no statistically significant differences were observed in clinical and demographic outcome measures between both groups (GCC and PSCC) upon study inclusion.

On day 7 after inclusion in the study, 23 out of 73 patients $(31 \%)$ had a GCC. Of these, $73 \%$ required AMV and had $\mathrm{FIO}_{2}>50 \%$, and a mean OI of 13 (standard deviation [SD]: \pm 13 ); 83\% required inotropes and had a mean VIS of 24 (SD: $24 \pm 38) ; 11 \%$ required $\mathrm{ECMO}$, and $86 \%$ met the criteria for MODS.

The overall mortality was $7 \%$ (5/73). After 72 hours of study inclusion, 5 patients in the group

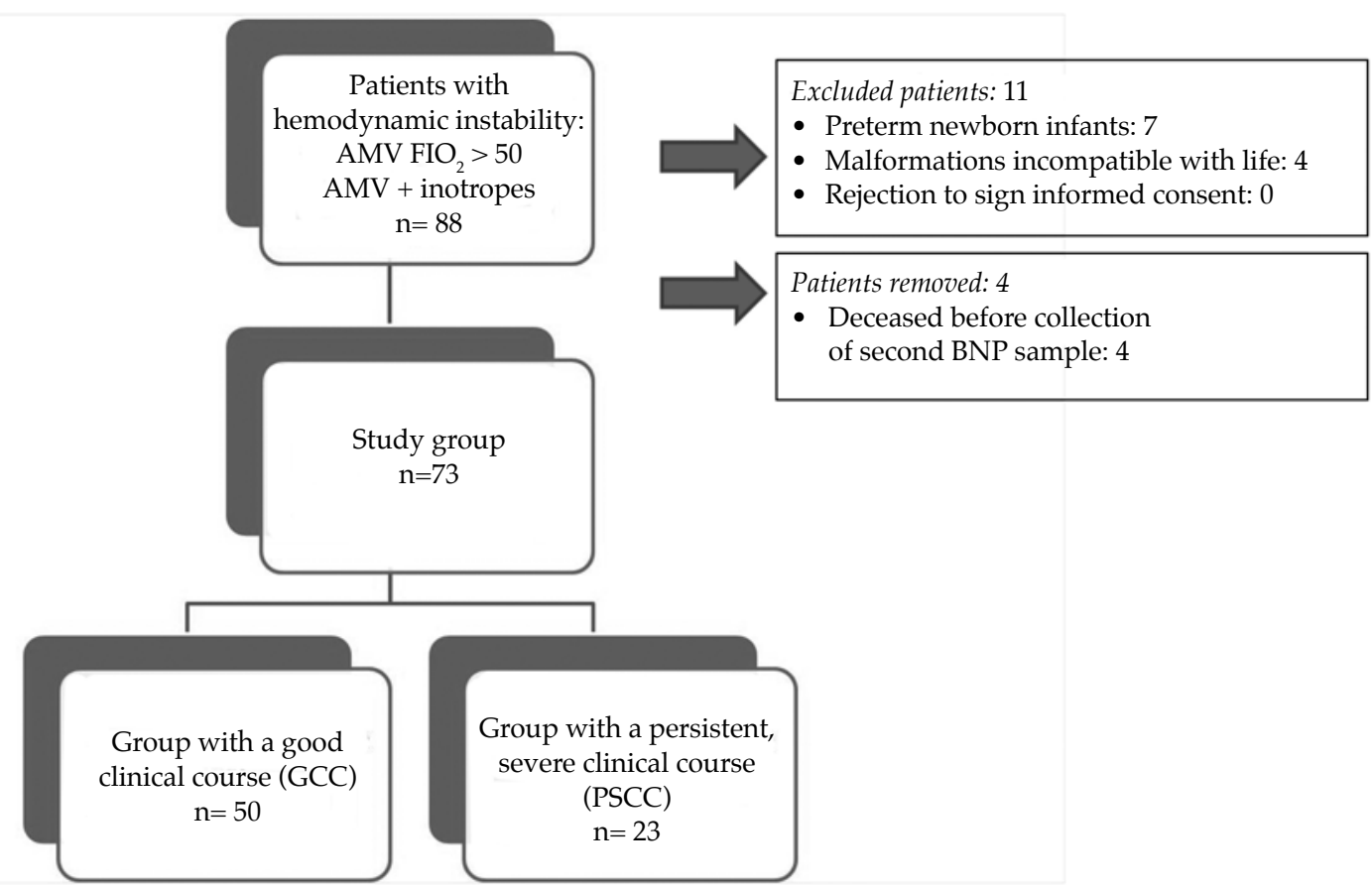

AMV: assisted mechanical ventilation; FIO2: fraction of inspired oxygen; BNP: B-type natriuretic peptide. 
with a PSCC had died. Four died within the 72 hours of protocol initiation and were removed.

TABLE 1. Characteristics of patients included in the study

\begin{tabular}{ll}
\hline & $\mathbf{N}=\mathbf{7 3}$ \\
\hline Age in days* & $3(1-14)$ \\
Gestational age in weeks* $^{*}$ & $37(35-38)$ \\
Male sex & $64 \%$ \\
Birth weight* $^{*}$ & $2880 \mathrm{~g}(2120-3500)$ \\
C-section & $63 \%$ \\
Apgar score at 5 min & $9(7-9)$ \\
Referred from other healthcare facilities & $73 \%$ \\
Diagnoses & Respiratory \\
& condition (65\%) \\
& Heart condition (8\%) \\
& Sepsis (19\%) \\
& Other (8\%) \\
\hline
\end{tabular}

* Values expressed as median with its corresponding interquartile range.
The median baseline BNP level was similar among patients from both groups (GCC and PSCC) ( $p$ : 0.15). However, the median BNP level at 72 hours was higher among patients with a PSCC compared to those with a GCC ( $p: 0.005)$ (Table 3).

Among all patients in the group with PSCC, the $\triangle B N P$ was positive, i.e., the BNP level at 72 hours was higher than at baseline. On the contrary, NBIs with a GCC showed a negative $\triangle B N P$. Such differences were statistically significant ( $p$ : 0.0002) (Tables 3 and 4).

The best cut-off point for $\triangle \mathrm{BNP}$ was +11 with a sensitivity of 0.87 and a specificity of 0.86 (PPV: 0.74; NPV: 0.93) (Table 5).

Figure 2 shows the ROC curve for $\triangle \mathrm{BNP}$ relative to patients' clinical course on day 7 ; the area under the curve was $0.86(0.76-0.97)$.

TABLE 2. Comparison of population upon admission, by clinical course

\begin{tabular}{|c|c|c|c|}
\hline & Good clinical course N: 50 & PSCC at 7 days N: 23 & $P$ \\
\hline $\begin{array}{l}\text { Age (days) } \\
\text { M (IQR) }\end{array}$ & $3(13)$ & $6(17)$ & 0.09 \\
\hline $\begin{array}{l}\text { Weight }(\mathrm{g}) \\
\bar{X}(\mathrm{SD})\end{array}$ & $2818(836)$ & $2487(1101)$ & 0.16 \\
\hline $\begin{array}{l}\text { Gestational age (weeks) } \\
\text { M (IQR) }\end{array}$ & $37(2)$ & $38(1.5)$ & 0.09 \\
\hline $\begin{array}{l}\text { Male sex } \\
\mathrm{n}(\%)\end{array}$ & $32(64 \%)$ & $15(65 \%)$ & 0.92 \\
\hline $\begin{array}{l}\mathrm{PHT}^{*} \\
\mathrm{n}(\%)\end{array}$ & $22(44 \%)$ & $9(39 \%)$ & 0.69 \\
\hline $\begin{array}{l}\text { Apgar score at } 5 \mathrm{~min} \\
\mathrm{X} \text { (SD) }\end{array}$ & $8.2(1.8)$ & $7.6(0.7)$ & 0.09 \\
\hline $\begin{array}{l}\text { SNAPPE } \\
X(\text { SD) }\end{array}$ & $30(22)$ & $29(19)$ & 0.99 \\
\hline $\begin{array}{l}\text { Lactic acid }(\mathrm{mmol} / \mathrm{L}) \\
\text { X (SD) }\end{array}$ & $3.5(3.7)$ & $3.5(4.1)$ & 0.76 \\
\hline $\begin{array}{l}\mathrm{FIO}_{2}^{* *}>50 \% \\
\mathrm{n}(\%)\end{array}$ & $39(78 \%)$ & $17(74 \%)$ & 0.70 \\
\hline $\begin{array}{l}\text { Oxygenation index } \\
\text { X (SD) }\end{array}$ & $12(9)$ & $12(11)$ & 0.59 \\
\hline $\begin{array}{l}\text { Inotropes } \\
\mathrm{n}(\%)\end{array}$ & $39(78 \%)$ & $16(70 \%)$ & 0.43 \\
\hline $\begin{array}{l}\text { Inotropic index } \\
X(S D)\end{array}$ & $25(38)$ & $27(65)$ & 0.18 \\
\hline $\begin{array}{l}\text { Pulmonary vasodilators } \\
\mathrm{n}(\%)\end{array}$ & $17(34 \%)$ & $9(39 \%)$ & 0.21 \\
\hline $\begin{array}{l}\text { Nitric oxide (iNO) } \\
\mathrm{n}(\%)\end{array}$ & $13(26 \%)$ & $3(13 \%)$ & 0.21 \\
\hline $\begin{array}{l}\text { Impairment of renal function } \\
\mathrm{n}(\%)\end{array}$ & $6(12 \%)$ & $3(13 \%)$ & 0.9 \\
\hline $\begin{array}{l}\text { Impairment of liver function } \\
\mathrm{n}(\%)\end{array}$ & $11(22 \%)$ & $5(21 \%)$ & 0.98 \\
\hline $\begin{array}{l}\text { Multiple organ dysfunction syndrome } \\
\mathrm{n}(\%)\end{array}$ & $44(88 \%)$ & $18(78 \%)$ & 0.28 \\
\hline
\end{tabular}

Values are expressed as average $(\overline{\mathrm{X}})$ and standard deviation $(\mathrm{SD})$ or as median $(\mathrm{M})$ and interquartile range (IQR).

* PHT: pulmonary hypertension. ${ }^{* *} \mathrm{FIO}_{2}$ : fraction of inspired oxygen. PSCC: persistent, severe clinical course.

SNAPPE: Score for Neonatal Acute Physiology-Perinatal Extension. 


\section{DISCUSSION}

BNP modulates biological processes through the activation of natriuretic peptide receptor-A (NPR-A), and is a fast and highly sensitive marker of myocardial dysfunction. ${ }^{23}$ The production ratio of BNP and NT-proBNP, its inactive precursor, is $1: 1$, so they may be equally useful and can be used based on their availability in each facility. ${ }^{24}$

Our study demonstrated the usefulness of measuring BNP in critically-ill NBIs to predict their clinical course in the short term.

Although the baseline BNP level on admission was not associated with the patient's prognosis, sequential determinations showed that a reduced BNP level in the second sample correlated with GCC whereas an increased BNP level was related to an unfavorable clinical course in the short term.

The low discrimination power of BNP measurement on admission, as an isolated value, had also been observed by other authors, ${ }^{34}$ who reported that serial determinations of NT-proBNP were better prognostic predictors than an isolated measurement. However, other authors have reported an adequate correlation between the measurement of BNP on the first day of life of NBIs with diaphragmatic hernia and their clinical course. $^{35}$
The wide dispersion of BNP levels observed in the first week of life shows the important role played by BNP during transition to extrauterine life. ${ }^{7}$ In our study, the isolated baseline BNP value also exhibited a wide dispersion among patients.

BNP has been extensively studied in patients with congenital heart disease; it has been demonstrated that BNP was higher in this population than in control individuals, including clinically stable patients. ${ }^{12,13,25}$

It is also a useful tool to identify in advance patients who, following a corrective surgery for heart malformations, will develop heart failure, and also as an indicator of the right time for the procedure. ${ }^{26,27}$

BNP measurements in preterm NBIs showed a strong correlation between an increased BNP and the presence of hemodynamically significant ductus arteriosus. ${ }^{20}$

There are reports about the capability of BNP to differentiate respiratory distress syndrome from PHT in NBIs; this means that BNP measurement may tell the difference between patients with a purely respiratory condition and those with hemodynamic or cardiac compromise. ${ }^{28} \mathrm{~A}$ strong correlation has been observed, in NBIs with PHT, among BNP,

TABLE 3. Comparison of B-type natriuretic peptide levels at 0 and 72 hours and delta B-type natriuretic peptide value as per the patients' clinical course

\begin{tabular}{lcccc}
\hline & ${ }^{*}$ TOTAL $\mathbf{n}=\mathbf{7 3}$ & ${ }^{* *}$ GCC $\mathbf{n}=\mathbf{5 0}$ & ${ }^{* * *}$ PSCC $\mathbf{n}=\mathbf{2 3}$ & $\boldsymbol{p}$ \\
\hline BNP 0 h & $459(185 / 1555)$ & $498(196 / 2297)$ & $378(92 / 1238)$ & 0.15 \\
BNP 72 h & $360(91 / 1165)$ & $271(68 / 737)$ & $1386(136 / 3760)$ & 0.005 \\
$\triangle$ BNP & $-265($ DE 2494) & $-967(-1656 /-278)$ & $1260(426 / 2094)$ & 0.0002 \\
\hline
\end{tabular}

BNP values are expressed as pg/mL. Values are expressed as median (IQR: 25-75).

$\triangle B N P$ values are expressed as median (SD). * Total: all patients included in the study.

${ }^{* *}$ GCC: good clinical course. ${ }^{* * *}$ PSCC: persistent, severe clinical course.

TABLE 4. Result of variations in B-type natriuretic peptide among studied patients and its relationship to their clinical course

\begin{tabular}{lccc}
\hline & ${ }^{\mathbf{1}}$ GCC $\mathbf{n}=\mathbf{2 3}$ & ${ }^{2}$ PSCC $\mathbf{n}=\mathbf{5 0}$ & Total $\mathbf{n}=\mathbf{7 3}$ \\
\hline${ }^{*} \Delta \mathrm{BNP}+$ & 20 & 7 & 27 \\
${ }^{* *} \Delta \mathrm{BNP}-$ & 3 & 43 & 46 \\
\hline
\end{tabular}

${ }^{*}$ Delta-BNP +: patients with an increased BNP level

in their second sample compared to the first.

** Delta-BNP -: patients with a reduced BNP level

in their second sample compared to the first.

${ }^{1}$ PSCC: persistent, severe clinical course.

${ }^{2}$ GCC: good clinical course.
TABLE 5. Result of variations in B-type natriuretic peptide among patients and its capability to predict their clinical course

\begin{tabular}{lcc}
\hline & & 95\% CI \\
\hline Sensitivity & $87.0 \%$ & $67.9-95.5 \%$ \\
Specificity & $86.0 \%$ & $73.8-93.0 \%$ \\
Positive predictive value & $74.1 \%$ & $55.3-86.8 \%$ \\
Negative predictive value & $93.5 \%$ & $82.5-97.8 \%$ \\
False positive ratio & $14.0 \%$ & $7.0-26.2 \%$ \\
False negative ratio & $13.0 \%$ & $4.5-32.1 \%$ \\
Accuracy & $86.3 \%$ & $76.6-92.4 \%$ \\
Youden index $(\mathrm{J})$ & 0.7 & \\
\hline
\end{tabular}

CI: confidence interval. 
clinical severity, ${ }^{29,30}$ and treatment response. ${ }^{31}$

Baptista et al. ${ }^{32}$ studied BNP levels in patients with congenital diaphragmatic hernia (CDH) and observed an association among BNP levels, the severity of PHT, and mortality in this population. Other authors have reported that serial measurements of BNP levels among NBIs with $\mathrm{CDH}$ predicted the severity of PHT and ECMO requirement, ${ }^{33}$ which was consistent with our findings.

Based on them, it may be stated that an increase in BNP levels obtained from 2 consecutive samples collected 72 hours apart predicts PSCC whereas a reduction predicts GCC in the short term. Likewise, it may be stated that such difference can be useful as a short-term prognostic tool.

An increase in BNP levels measured sequentially in critically-ill patients hospitalized in the NICU may predict hemodynamic changes, prognosis and/or response to treatment.

One of the limitations of our study is that no correlation was established between patients' BNP levels and echocardiograms, as reported in other studies. ${ }^{34,35}$ Likewise, it is necessary to study whether these outcomes are reproducible in other facilities.

Further studies are required to support the usefulness of BNP measurements as a predictor of clinical course among critically-ill NBIs.

To conclude, BNP measurements in a population of NBIs with cardiorespiratory instability, as per our results, can predict their clinical course in the short term. It is a sensitive, readily available test that can be easily performed in many healthcare facilities, and is especially aimed for those sites where there is not a tertiary care neonatal intensive care unit because it makes it possible to know, in advance, whether it is necessary to transfer a patient to a facility with a higher level of care.

\section{Acknowledgments}

The authors would like to thank the parents of patients who agreed to help develop scientific knowledge, and the physicians and nurses from the neonatal intensive care unit of Hospital de Pediatría "Prof. Dr. Juan P. Garrahan" for their significant dedication to the development of this project.

\section{REFERENCES}

1. Bar-Oz B, Lev-Sagie A, Arad I, et al. N-terminal pro-B type natriuretic peptide concentrations in mothers just before delivery, in cord blood and newborns. Clin Chem 2005;51(5):926-7.

2. Bakker J, Gies I, Slavenburg B, et al. Reference values for N-terminal pro-B-typenatriuretic peptide in umbilical cord blood. Clin Chem 2004;50(12):2465.

3. Fortunato G, Carandente Giarrusso P, Martinelli P, et al. Cardiac troponin $\mathrm{T}$ and amino-terminal pro-natriuretic peptide concentrations in fetuses in the second trimester and in healthy neonates. Clin Chem Lab Med 2006;44(7):834-6.

4. El-Khuffash A, Molloy E. Are B-type natriuretic peptide (BNP) and N-terminal-pro-BNP useful in neonates? Arch Dis Child Fetal Neonatal Ed 2007;92(4):F320-4.

5. Hammerer-Lercher A, Neubauer E, MullerS, et al.Head-tohead comparison of $\mathrm{N}$-terminal pro-brainnatriureticpeptide, brainnatriuretic peptideand $\mathrm{N}$-terminal pro-atrial natriuretic peptide in diagnosing left ventricular dysfunction. Clin Chim Acta 2001;310(2):193-7.

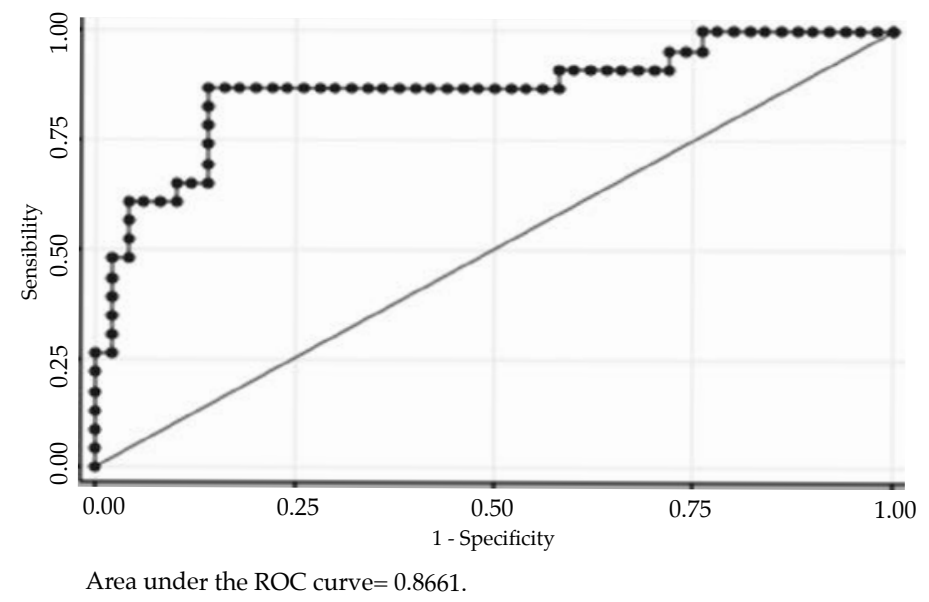

ROC curve: receiver operating characteristic curve. 
6. Vijlbrief, Manon J, Hans K, et al. Use of cardiac biomarkers in neonatology. Pediatr Res 2012;72(4):337-43.

7. Hammerer-Lercher A, Mair J, Tews G, et al. N-terminal pro-B-tipe natriuretic peptide concentrations are markedly higher in the umbilical cord blood of newborns than in their mothers. Clin Chem 2005;51(5):913-5.

8. Johns M, Stephenson C. Amino-Terminal Pro B type Natriuretic Peptide testing in Neonatal and Pediatric patients. Am J Cardiol 2008;101(3A):A76-81.

9. Nir A, Bar-Oz B, Perles Z, et al. N-terminal pro-B-Type natriuretic peptide: reference plasma levels from birth to adolescence: elevated levels at birth and in infants and children with heart diseases. Acta Paediatr 2004;93(5):603-7.

10. Cohen S, Springer C, Avital A, et al. Amino Terminal pro brain natriuretic peptide: heart or lung disease in pediatric respiratory distress. Pediatrics 2005;115(5):1347-50.

11. Wang F, Wu Y, Tang L, et al. Brain Natriuretic peptide for prediction of mortality in patients with sepsis: a systematic review and meta-analysis. Crit Care 2012;16(3):R74.

12. Nasser N, Perles Z, Rein AJ, et al. NT-pro BNP as a marker for persistent cardiac disease in children with history of dilated cardiomyopathy and myocarditis. Pediatr Cardiol 2006;27(1):87-90.

13. Dodge-Khatami A, Büchel EV, Knirch W, et al. Brain natriuretic peptide and magnetic resonance imaging in tetralogy with right ventricular dilatation. Ann Thorac Surg 2006;82(3):983-8.

14. Tulevski H, Groenink M, van Der Wall EE, et al. Increased brain and atrial natriuretic peptides in patients with chronic right ventricular pressure overload: correlation between plasma neurohormones and right ventricular dysfunction. Heart 2001;86(1):27-30.

15. Bernus A, Wagner BD, Accurso F, et al. Brain natriuretic peptide levels in managing pediatric patients with pulmonary arterial hypertension. Chest 2009;135(9):745-51.

16. Choi BM, Lee KH, Eun BL, et al. Utility of rapid B-type natriuretic peptide assay for diagnosis of symptomatic patent ductus arteriosus in preterm infants. Pediatrics 2005;115(3):e255-61.

17. Flynn P, Da Graca R, Auld P, et al. The use of bedside assay for plasma B-type natriuretic peptide as a biomarker in the management of patent ductus arteriosus in premature neonates. J Pediatr 2005;147(1):38-42.

18. Chen S, Tacy T, Clyman R. How useful are B-type natriuretic peptide measurements for monitoring changes in patent ductus arteriosus shunt magnitude? J Perinatol 2010;30(12):780-5.

19. Hsu JH, Yang SN, Chen HL, et al. B-type natriuretic peptide predicts responses to indomethacin in premature neonates with patent ductus arteriosus. J Pediatr 2010;157(1):79-84.

20. Lu KS, Guo XL, Lv JF, et al. Value of amino-terminal pro-brain natriuretic peptide as a predictive marker of symptomatic patent ductus arteriosus in preterm infants. Zhongguo Dang Dai Er Ke Za Zhi 2015;17(11):1160-4.

21. El-Khuffash A, Barry D, WalshK, etal. Biochemical markers may identify preterm infants with a patent ductus arteriosus at high risk of death or severe intraventricular haemorrhage. Arch Dis Child Fetal Neonatal Ed 2008;93(6):F407-12.

22. Gaies M, Jeffries H, Niebler R, et al. Vasoactive-Inotropic Score is Associated with Outcome After Infant Cardiac Surgery: An Analysis from the Pediatric Cardiac Critical Care Consortium and Virtual PICU System Registries. Pediatr Crit Care Med 2014;15(6):529-37.

23. Calzetta L, Orlandi A, Page C, etal. Brain natriuretic peptide: Much more than a biomarker. Int J Cardiol 2016;221:1031-8.

24. Holmström H, Hall C, Thaulow E. Plasma levels of natriuretic peptides and hemodynamic assessment of patent ductus arteriosus in preterm infants. Acta Paediatr 2001;90(2):184-91.

25. Cowley GC, Bradley JD, Shaddy RE. B-type natriuretic peptide levels in congenital heart disease. Pediatr Cardiol 2004;25(4):336-40.

26. Norozi K, Buchhorn R, Bartmus D, et al. Elevated brain natriuretic peptide and reduced exercise capacity in adult patients operated on for tetralogy of Fallot is due to biventricular dysfunction as determined by myocardial performance index. Am I Cardiol 2006;97(9):1377-82.

27. Weber M, Arnold R, Rau M, et al. Relation of N-terminal Pro-B natriuretic peptide to progression of aortic valve disease. Eur Heart J 2005;26(10):1023-30.

28. Reynolds EW, Ellington JG, Vranicar M, et al. Brain-type natriuretic peptide in the diagnosis and management of persistent pulmonary hypertension of the newborn. Pediatrics 2004;114(5):1297-304.

29. Vijlbrief DC, Benders MJ, Kemperman H, et al. B-type natriuretic peptide and rebound during treatment for persistent pulmonary hypertension. J Pediatr 2012;160(1):111-5.e1.

30. Shah N, Natarajan G, Aggarwal S. B-type natriuretic peptide: biomarker of persistent pulmonary hypertension of the newborn? Am J Perinatol 2015;32(11):1045-9.

31. Treggani M, Bendyelid K, Yanez D, et al. Atrial and brain natriuretic peptide concentrations and the response to inhaled nitric oxide in patients with acute respiratory distress syndrome. J Crit Care 2010;25(1):23-9.

32. Baptista M, Rocha G, Clemente F, et al. N-Terminal-pro-B Type Natriuretic Peptide as a Useful Tool to Evaluate Pulmonary Hypertension and Cardiac Function in $\mathrm{CDH}$ Infants. Neonatology 2008;94(1):22-30.

33. Partridge EA, Hanna BD, Rintoul NE, et al. Brain-type natriuretic peptide levels correlate with pulmonary hypertension and requirement for extracorporeal membrane oxygenation in congenital diaphragmatichernia. J Pediatr Surg 2015;50(2):263-6.

34. Rusconi PG, Ludwig DA, Ratnasamy C, et al. Serial measurements of serum NT-proBNP as markers of left ventricular systolic function and remodeling in children with heart failure. Am Heart J 2010;160(4):776-83.

35. Steurer M, Moon-Grady A, Fineman J, et al. B-type natriuretic peptide: a prognostic marker in congenital diaphragmatic hernia. Pediatr Res 2014;76(6):549-54. 